Trinity University

Digital Commons @ Trinity

Chemistry Faculty Research

Chemistry Department

$1-16-2020$

\title{
Dual-Balance Electrodynamic Trap as a Microanalytical Tool for Identifying Gel Transitions and Viscous Properties of Levitated Aerosol Particles
}

\author{
David S. Richards \\ Trinity University, drichar2@trinity.edu \\ Kristin L. Trobaugh \\ Trinity University, ktrobaug@trinity.edu \\ Josefina Hajek-Herrera \\ Trinity University, jhajekhe@trinity.edu \\ Ryan D. Davis \\ Trinity University, rdavis5@trinity.edu
}

Follow this and additional works at: https://digitalcommons.trinity.edu/chem_faculty

Part of the Chemistry Commons

\section{Repository Citation \\ Richards, D.S., Trobaugh, K.L., Hajek-Herrera, J., \& Davis, R.D. (2020). Dual-balance electrodynamic trap as a microanalytical tool for identifying gel transitions and viscous properties of levitated aerosol particles. Analytical Chemistry. doi: 10.1021/acs.analchem.9b04487}

This Article is brought to you for free and open access by the Chemistry Department at Digital Commons @ Trinity. It has been accepted for inclusion in Chemistry Faculty Research by an authorized administrator of Digital Commons@ Trinity. For more information, please contact jcostanz@trinity.edu. 


\title{
Dual-Balance Electrodynamic Trap as a Microanalytical Tool for Identifying Gel Transitions and Viscous Properties of Levitated Aerosol Particles
}

\author{
David S. Richards, Kristin L. Trobaugh, Josefina Hajek-Herrera, and Ryan D. Davis* \\ Cite This: https://dx.doi.org/10.1021/acs.analchem.9b04487 \\ Read Online
}

ACCESS

Llll Metrics \& More

Article Recommendations

Supporting Information

ABSTRACT: The formation of gelatinous networks within an aerosol particle significantly alters the physicochemical properties of the aerosol material. Existing techniques for studying gel transitions rely on bulk rheometry, which is limited by contact with the sample, or microrheological techniques such as holographic optical tweezers, which rely on expensive equipment and high-powered lasers that can degrade light-absorbing aerosol. Here, we present a new technique to probe the microrheological characteristics of aerosol particles and explore gel formation under atmospheric conditions in a contactless environment without the need for high-power light sources. In a dualbalance quadrupole electrodynamic balance, levitated droplets of

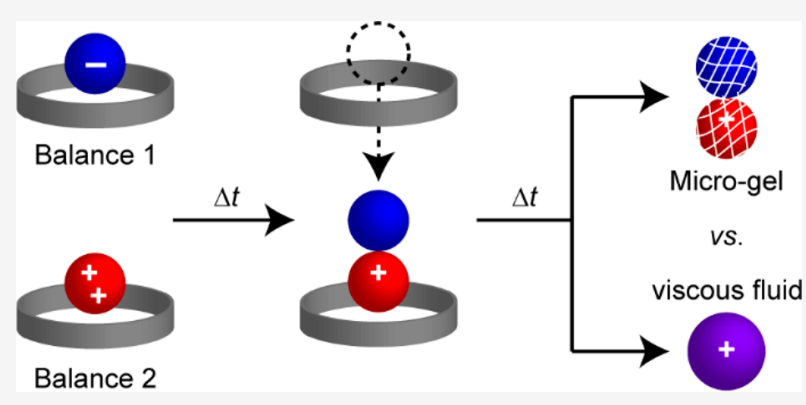
opposite polarity are trapped and equilibrated at fixed relative humidity $(\mathrm{RH})$ and then subsequently merged, and the physical characteristics of the merged droplets are monitored as a function of time and $\mathrm{RH}$ using imaging techniques. By comparing the $\mathrm{RH}$ dependent characteristics of $\mathrm{MgSO}_{4}$ (known to undergo a gel transition) to glucose and sucrose (known to remain as viscous Newtonian fluids) under fixed equilibration time scales, we demonstrate that gel phase transitions can be identified in aerosol particles, with $\mathrm{MgSO}_{4}$ abruptly transitioning to a rigid microgel at $30 \% \mathrm{RH}$. Further, we demonstrate this technique can be used to also measure aerosol viscosity and identify non-Newtonian fluid dynamics in model sea spray aerosol composed of $\mathrm{NaCl}_{1} \mathrm{CaCl}_{2}$, and sorbitol. Thus, using this experimental technique, it is possible to distinguish between aerosol compositions that form viscous Newtonian fluids and those that undergo a gel transition or form non-Newtonian fluids. This technique offers a simple and costeffective analytical tool for probing gel transitions outside of bulk solubility limits, with relevant applications ranging from atmospheric science to microengineering of soft matter materials.

\begin{abstract}
A morphous phase transitions in microenvironments are of significant interest to a wide variety of disciplines from pharmacokinetics in living systems to atmospheric and environmental science. ${ }^{1,2}$ In particular, gel transitions within aerosol particles are of interest in atmospheric science and industrial spray-drying and microencapsulation processes. ${ }^{3,4}$ In the atmosphere, the viscosity and phase state of aerosols influence climate and air quality by impacting, for example, aerosol reactivity and hygroscopicity. ${ }^{4-7}$ Specifically, diffusion of particle-phase water and reactive compounds can be extremely hindered in ultraviscous and gelatinous aerosol states. ${ }^{4-7}$ It has been shown that these diffusive limitations influence the rate of chemical processes and thus particle lifetime. ${ }^{5}$ Viscous Newtonian fluids and semisolid gelatinous states have distinct and different relative humidity $(\mathrm{RH})$-dependent diffusion limitations. ${ }^{7}$ Thus, distinguishing between viscous fluids and semisolid gels is essential to accurately representing the aerosol effect on climate and air quality.

Despite the fundamental interest in gel transitions, there is no comprehensive understanding of gel formation in the aerosol phase. ${ }^{6}$ In part, this limited understanding is because aerosol
\end{abstract}

particles frequently exist outside of bulk solubility limits. ${ }^{7-9}$ Thus, exploring gel transitions of atmospherically relevant compounds with bulk rheometry is not possible across the full range of supersaturation levels accessible in the atmosphere or within aerosol sprays. For example, aqueous $\mathrm{MgSO}_{4}$, which crystallizes under bulk conditions with increasing supersaturation, readily forms a semisolid gel in microdroplets. ${ }^{7}$ Additionally, $\mathrm{NaCl}$-sodium dodecyl sulfate (SDS) microgels form in aerosol particles at SDS concentrations inaccessible in bulk. ${ }^{9}$ Gels are frequently non-Newtonian fluids, i.e., their apparent viscosity is not constant at constant temperature. ${ }^{6,10}$ Gels and other non-Newtonian fluids are therefore not necessarily expected to have a single dynamic viscosity and can behave mechanically like solids under low shear while behaving like a liquid at high shear. Other non-Newtonian fluids, such as

Received: October 1, 2019

Accepted: January 16, 2020

Published: January 16, 2020 

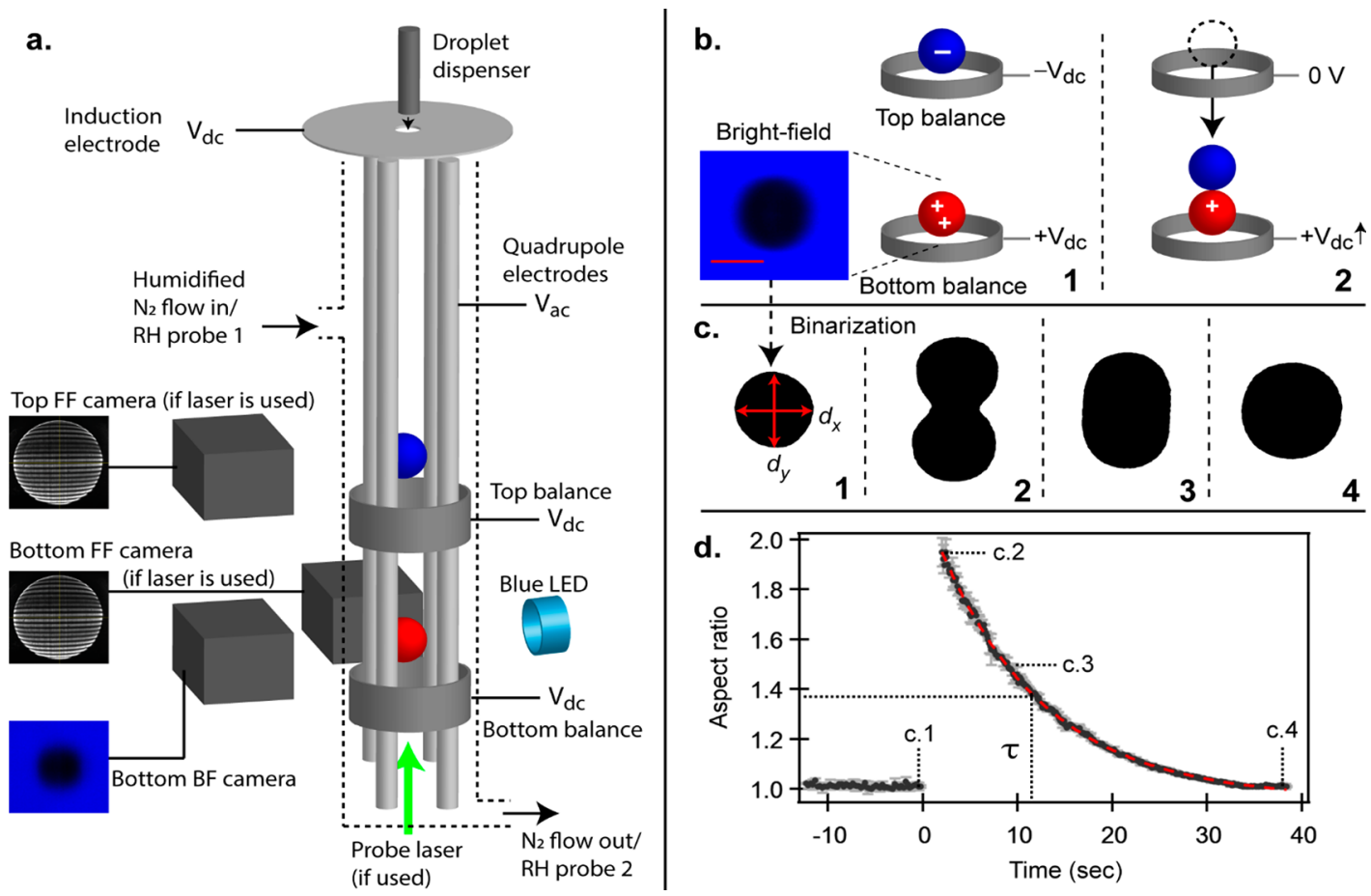

Figure 1. (a) Overview of the experimental arrangement. Note that the probe laser is not necessary for levitation nor for imaging of merged droplets; the laser and far-field (FF) imaging are complementary tools that are available but not required. (b) The process for merging droplets. As shown in panel 1, droplets of opposite polarity are simultaneously levitated at a constant $\mathrm{RH}$ for a fixed equilibration time ( 5 min for exploring gel formation; 30 min or more when measuring aerosol viscosity). Scale bar is $30 \mu \mathrm{m}$. As shown in panel 2, droplets are subsequently merged and the bottom balance is adjusted to compensate for increase in mass and partial charge neutralization. (c) Once merged, bright-field (BF) images are binarized, and the aspect ratio is tracked as a function of time. (d) A plot of aspect ratio as a function of time for a merging event of binary glucose droplets at $26 \% \mathrm{RH}$. The corresponding binarized images from part $\mathrm{c}$ are indicated on the graph. The red dashed line indicates the exponential fit $(\tau=11.6 \mathrm{~s})$. Errors bars are \pm 1 $\mathrm{SD}$ of five processed images.

colloidal suspensions, may exhibit time-dependent viscosity. ${ }^{11}$ Such non-Newtonian behavior complicates predictions of aerosol properties, including rates of the particle-phase diffusion of water. ${ }^{7}$ Ambient aerosol can also exist as inhomogeneous, internally mixed solid-liquid mixtures. ${ }^{4}$ Thus, to explore gelation of atmospheric aerosol and the formation of inhomogeneous mixtures, it will be important to overcome the limitations of bulk rheometry while utilizing techniques that are sensitive to non-Newtonian fluid dynamics.

To overcome the limits of bulk rheometry, a number of complementary aerosol-based microrheological techniques have been introduced, each with their own distinct advantages and limitations. The electrical low-pressure impactor technique assesses particle phase based on bounce factor but relies on substrate deposition and indirectly infers liquid vs nonliquid aerosol. ${ }^{12,13}$ Recently, an atomic force microscopy (AFM)based technique has been introduced that qualitatively distinguishes between liquid, semisolid, and solid states but is also predicated on substrate deposition. ${ }^{14-16}$ Viscosity and phase have also been studied with the bead-mobility and pokeflow techniques but depend on substrate deposition. ${ }^{17-19}$ The dimer coagulation, isolation, and coalescence (DCIC) technique requires no substrate deposition and can perform measurements on submicrometer aerosol but can access a limited viscosity range. ${ }^{20}$ Holographic optical tweezers (HOT) is another technique that can explore aerosol phase of levitated aerosol $^{9,21-23}$ and is a microrheological technique that is sensitive to the non-Newtonian fluid dynamics that are frequently associated with semisolid gels.
Despite the many advantages of the HOT technique and optical trapping, in general, there are several drawbacks associated with optical-tweezing methods of aerosols that hinder their application for broadly exploring gel formation. Most notably, the potential absorption of laser light can limit applicability. For example, marine organic material is known to absorb visible light, ${ }^{24}$ and marine microgel formation is sensitive to light exposure and temperature. ${ }^{25}$ Therefore, for marine aerosol species that absorb light at the trapping laser wavelength, the absorption of laser light can be potentially consequential in the analysis of gel phase transitions due to potential disruption of polymer-cross-linking within the aerosol through an inevitable increase in temperature from laserinduced heating. Thus, to fully characterize the aerosol phase and gel formation of atmospherically relevant aerosol compositions, new techniques are necessary that are sensitive to non-Newtonian fluid dynamics but do not require a trapping laser.

Here, we demonstrate a new microrheological tool and experimental method for identifying aerosol gel phase transitions using electrostatic levitation techniques via a dualbalance linear quadrupole electrodynamic balance (DBQ-EDB, Figure 1a). In this design, laser light is not required for particle trapping. Simultaneous equilibration of two particles is made possible through the inclusion of two counterbalance electrodes positioned vertically such that particles of opposite surface charge, equilibrated under the same conditions, can be subsequently merged, and viscosity and phase state can be inferred through bright-field imaging. While electrostatic levitation has been widely used as a method for analysis of, 

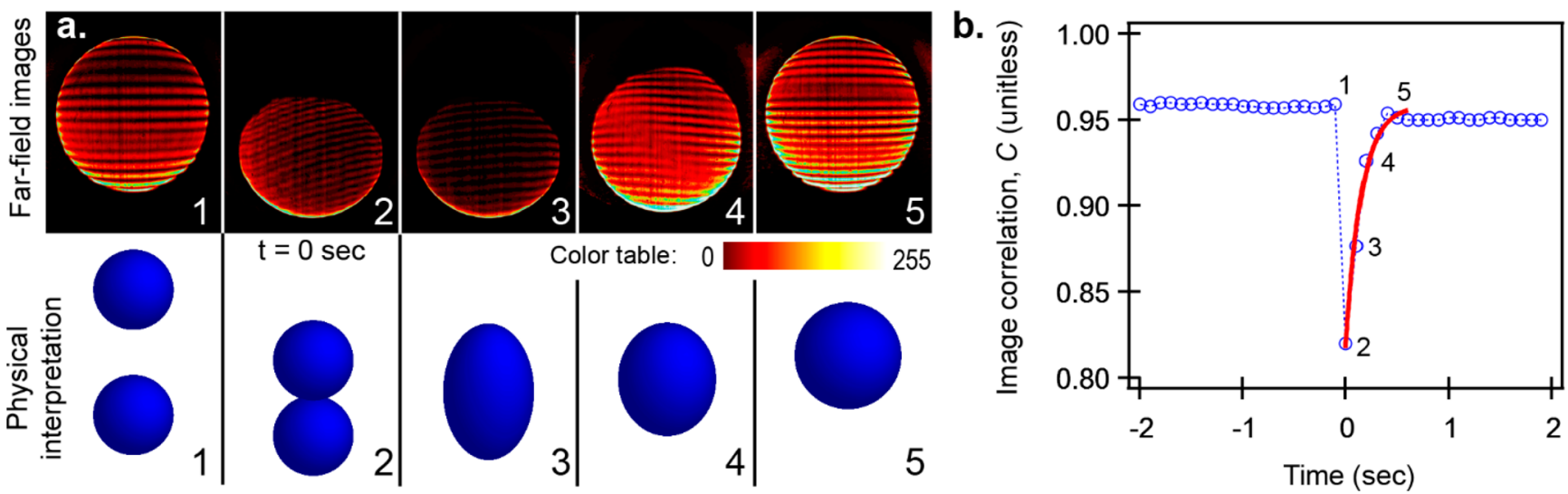

Figure 2. (a) Top panel: far-field images collected during a merging event of binary glucose at $38 \% \mathrm{RH}$. Bottom panel: illustrated representation of the merging event. (b) A plot of image correlation, $C$, as a function of time for the merging event at $38 \% \mathrm{RH}$. The numbers indicated on the plot correspond to the far-field images from part a. The solid red line is the exponential fit.

e.g., aerosol hygroscopicity ${ }^{26}$ and reaction kinetics, ${ }^{27}$ to our knowledge, it has not been previously used as a microrheological technique. Using this method, we show stability and control over $\mathrm{RH}$ and particle trapping capability on an indefinite time scale. We demonstrate the capability of the DBQ-EDB technique to accurately determine aerosol viscosity (of sorbitol and aqueous glucose), identify gel transitions (of $\mathrm{MgSO}_{4}$ ), and detect nonNewtonian fluid dynamics (of solid-liquid model sea spray mixtures composed of sorbitol, $\mathrm{NaCl}$, and $\mathrm{CaCl}_{2}$ ).

\section{EXPERIMENTAL SECTION}

Electrostatic Levitation. The experimental arrangement for the DBQ-EDB is shown in Figure 1a. The DBQ-EDB is similar in design to linear quadrupole traps described else-

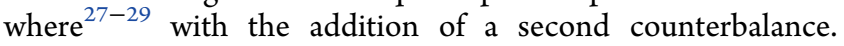
Droplets were generated using piezoelectric droplet dispensers with a $50 \mu \mathrm{m}$ diameter orifice (MicroFab MJ-APB-050) from 5 wt $\%$ aqueous stock solutions. Droplets were injected into the DBQ-EDB through an induction electrode ( $<400 \mathrm{~V}$ typically) that induced a net charge on the droplets and allowed them to be confined axially within the electrostatic field of the quadrupole ( $V_{\mathrm{AC}}, 600 \mathrm{~V}$ typical amplitude, $300 \mathrm{~Hz}$ typical frequency) and supported vertically by the counterbalance electrodes $(< \pm 500$ $\mathrm{V}_{\mathrm{DC}}$ typical). The dual-balance system was designed with two counterbalance electrodes to counter gravity and the humidified nitrogen flow, and thus two charged particles could be levitated simultaneously, one $\sim 5 \mathrm{~cm}$ above the other. The magnitude of the charge induced on the droplet levitated in the top balance was adjusted to be less than the particle levitated in the bottom balance. By doing so, the merged dimer contained a net charge large enough to remain levitated in the bottom balance after merging, as illustrated in Figure $1 \mathrm{~b}$.

Environmental Control and Monitoring. All experiments were conducted at room temperature $(295 \pm 2 \mathrm{~K})$. The $\mathrm{RH}$ in the DBQ-EDB was controlled by varying the ratio of dry and humidified nitrogen gas flows (300 to $500 \mathrm{sccm}$ total flow). Calibrated RH sensors (Honeywell HIH-4602-C) were placed before and after the DBQ-EDB, and the $\mathrm{RH}$ within the chamber was taken as the average of the two sensors $( \pm 1 \mathrm{SD}, \sim 1 \% \mathrm{RH}$ typical).

Imaging of Merging Events and Determining Coalescence Time Scales. Bright-field imaging was used to determine the aspect ratio of merged droplets as a function of time at long time scales $(>3 \mathrm{~s})$, as shown in Figure $1 \mathrm{c}^{21-23}$
Droplets were backlit with a blue LED and imaged with a $20 \times$ long-working-distance microscope objective using a CMOS camera (max frame rate of $\sim 15 \mathrm{fps}$ ). Droplets were sized with bright-field images by calibrating the CMOS camera sensor. For Newtonian fluids, coalescence of the merged dimers is evident from the exponential relaxation of the dimer aspect ratio from $\sim 2$ back to 1, as shown in Figure 1d. This relaxation (for Newtonian fluids) occurs with a time constant $\tau$ that is directly proportional to the viscosity of the merged droplets.

Upon merging, droplets were momentarily removed from the field of view of the bright-field imaging camera due to the change in droplet size and surface charge, as demonstrated in Movie S1. This change in dimer position is entirely inadvertent and largely a result of increased gravitational force due to increased mass. The merged dimer was brought back into the field of view by increasing the counterbalance electrode voltage, a process which took $\sim 3 \mathrm{~s}$, during which time the merged dimer was not visible in the bright-field images. Thus, for rapid coalescence $(\tau<3 \mathrm{~s})$, droplets were illuminated with a $532 \mathrm{~nm}$ diode-pumped $\mathrm{Nd}$ :YAG laser, and the elastically scattered far-field light was imaged with a CMOS camera. Although far-field laser scatter imaging is not necessary for levitation nor to detect gel transitions (see Results and Discussion), the laser is a complementary tool that be can be utilized, if so desired, as shown in Figure 2. As seen here, disruptions in the far-field interference fringes of merged dimers were utilized to track coalescence at time scales from $\sim 0.1$ to $3 \mathrm{~s}$.

As demonstrated in Figure 2a, panel 1, when elastically scattered laser light is imaged in the far-field for a perfectly spherical droplet (i.e., premerging), linear interference fringes are visible that contain discrete maxima and minima. ${ }^{30}$ For a liquid droplet, the intensity distribution of the linear interference fringes is horizontally symmetric. As shown in Figure 2a, panels 2 to 4 , when droplets are merged, the merged dimer is no longer perfectly spherical, which creates a disruption in the horizontal symmetry of the linear interference fringes. When the merged droplets completely coalesce (i.e., return to a spherical shape), the horizontal symmetry in the linear interference fringes returns, as in Figure 2a, panel 5. These changes in the far-field images were thus quantified using an autocorrelation technique $^{30,31}$ to monitor the time scale of coalescence of droplets.

The images analysis is a template-based autocorrelation of the far-field images, where the template image was the original image 
offset in the horizontal $(x)$ direction, as described extensively elsewhere. ${ }^{30,31}$ Far-field images were recorded and then postprocessed in a custom LabVIEW program. An image within the recorded sequence was duplicated and then offset horizontally in the $x$-direction by $n$ pixels $(n=20$ pixels, typically). The absolute difference between overlapping pixel values of the offset image and the original image created a "defect" image (as shown in Figure S1). The average defect image intensity $\bar{I}_{\mathrm{D}}$ was normalized to the average far-field intensity $\bar{I}$ of the original image to calculate a correlation value $C$, given by eq 1 .

$$
C=\left(\bar{I}-\bar{I}_{\mathrm{D}}\right) / \bar{I}
$$

As shown in Figure 2b, for spherical droplets, the horizontal symmetry in the fringes is high, and thus, defect image intensity is low, and correlation values are high (with $C=1$ being the highest possible value). Upon merging, horizontal symmetry in the linear fringes is broken, and thus, the correlation value decreases. When the droplets completely coalesce, the correlation value returns to a higher, stable value. An exponential fit to a plot of $C$ as a function of time from the point of first contact provided $\tau$ for these cases.

Gels and non-Newtonian fluids are not necessarily expected to have a single dynamic viscosity and can behave mechanically like solids under low shear and therefore not coalesce. ${ }^{9}$ Thus, for gels and other non-Newtonian fluids, extracting a single $\tau$-value is not necessarily possible. In the cases where no coalescence was observed, the observation time over which the merged dimer remained rigid $\left(t_{\text {obs }}\right)$ was reported.

Materials. All chemicals were purchased from Sigma-Aldrich (>99\% purity) and used without further purification. Stock solutions were made from Millipore water $(18 \mathrm{M} \Omega)$ and filtered prior to dispensing.

Experimental Sequence for Exploring Gel Transitions. In the present study, time scales ( $\tau$ or observation time, $t_{\mathrm{obs}}$ ) were used to differentiate between $\mathrm{RH}$-dependent rheological behavior of glass-forming versus gel-forming systems, the former of which can occur gradually over a wide $\mathrm{RH}$ range, ${ }^{23}$ and the latter of which can undergo an abrupt phase transition where microstructural rearrangement rapidly occurs. ${ }^{7,32}$ Because the focus was to probe for an abrupt phase transformation (gelation), we therefore used a fixed equilibration time of 5 min for all experiments prior to merging droplets, analogous to the approach of using fixed residence times when probing ice nucleation or efflorescence. ${ }^{31}$ This fixed equilibration time limited the chances for obfuscating gel and glass transitions, i.e., because $\mathrm{RH}$-induced glass transitions require hours to days of equilibration, $^{23}$ a short equilibration time ensured observation of a glass transition would be unlikely and increased confidence that a gel transition had been observed. Furthermore, rather than estimating when a droplet had fully equilibrated to the ambient $\mathrm{RH}$ (which can take many hours at low $\mathrm{RH}$ ), ${ }^{23}$ a consistent equilibration time also limited variations in extracted $\tau$.

To perform an experiment at a constant $\mathrm{RH}$, two oppositely charged droplets $(30 \pm 2 \mu \mathrm{m}$ average diameter) were simultaneously levitated in the top and bottom balances at a constant RH and equilibrated for $5 \mathrm{~min}$, after which droplets were merged, and either far-field or bright-field images were collected. At long time scales, the laser was not used to avoid optical heating, if any. Images were postprocessed to determine $\tau$, if applicable. In cases where coalescence was not observed, merged dimers were levitated for a $t_{\mathrm{obs}}$ of $10^{4}$ to $10^{5} \mathrm{~s}$, and brightfield images were continually or intermittently collected. If no change in aspect ratio was observed over this time frame, a merging event was considered to have shown no coalescence.

Sequence for Determining Organic Aerosol Viscosity. To benchmark the system, we also measured the dry viscosity of sorbitol and the RH-dependent viscosity of binary glucose. While a fixed 5 min equilibration time was used to probe for gel transitions, longer equilibration times are necessary for accurate viscosity measurements. ${ }^{23}$ Thus, when viscosity was the intended measurement, droplet equilibration times of 30-60 min were used prior to merging. ${ }^{23}$ Droplets were assumed to be close to equilibrium when the DC voltage required to levitate the droplets stabilized (see Figure S2).

Safety. The DBQ-EDB utilizes high-voltage sources. All high-voltage was enclosed within the DBQ-EDB chamber.

\section{RESULTS AND DISCUSSION}

To demonstrate that the DBQ-EDB technique can be used to explore aerosol viscosity and identify gel transitions as well as non-Newtonian fluids, we benchmarked the technique by measuring the known viscosity of sorbitol and explored the $\mathrm{RH}$-dependent microrheology of binary $\mathrm{MgSO}_{4}$ (a known gelforming system). The microrheology of $\mathrm{MgSO}_{4}$ was compared to the RH-dependent rheology of Newtonian fluids. Binary glucose and sucrose are well-studied systems that are known to remain as Newtonian fluids and gradually vitrify ${ }^{18,23}$ and thus are used here as comparison to gel formation. Further, to explore other non-Newtonian fluids, we examined the coalescence of mixed solid-liquid droplets composed of sorbitol/ $\mathrm{CaCl}_{2}$ with $\mathrm{NaCl}_{(s)}$ inclusions. Because gels are characterized by a rigid semisolid structure and do not necessarily coalesce completely nor have a single associated viscosity, we present and discuss the microrheological results in terms of time scales $\left(\tau\right.$ or $\left.t_{\mathrm{obs}}\right)$, rather than viscosity, to distinguish gel-forming systems from viscous Newtonian fluids.

RH-Dependent Coalescence Time Scales. Newtonian Fluids Gradually Increase in Coalescence Time Scale with Decreasing $\mathrm{RH}$. The typical RH-dependent rheology of binary glucose is demonstrated in Figures $2 \mathrm{~b}$ and $1 \mathrm{~d}$ at $\sim 38$ and $26 \%$ $\mathrm{RH}$, respectively. As seen in Figure $2 \mathrm{~b}$, upon merging of binary glucose droplets at $38 \% \mathrm{RH}$, the dimer exponentially relaxes with a $\tau$-value of $0.15 \mathrm{~s}$. As seen in Figure $1 \mathrm{~d}$, upon decreasing the $\mathrm{RH}$ to $26 \% \mathrm{RH}, \tau$ increases by 2 orders of magnitude to $\sim 11.6 \mathrm{~s}$. In these examples, coalescence is complete and characterized by gradual, viscous flow that is consistent with the overdamped regime of a viscous Newtonian fluid, where $\tau$ is directly proportional to viscosity of the fluid. ${ }^{23}$ The increase in $\tau$ (and thus viscosity) with decreasing $\mathrm{RH}$ is due to decreasing water content. $^{23}$ As shown in Figure 3 for glucose, as the $\mathrm{RH}$ is decreased from $\sim 39 \pm 2$ to $17 \pm 2 \% \mathrm{RH}$, there is approximately 1 order of magnitude increase in $\tau$ for every $5-6 \%$ decrease in $\mathrm{RH}$, consistent with previous observations. ${ }^{18,23}$ Notably, this same trend is observed with both far-field and bright-field imaging techniques, demonstrating the validity of using far-field image analysis for monitoring droplet coalescence. For all merging events, complete coalescence was observed with an exponential relaxation from bispherical dimer to spherical droplet. If the premerging equilibration time is $60 \mathrm{~min}$, binary glucose still exhibits similar trends but shifted to a higher $\mathrm{RH}$, as seen in Figure S3. Sucrose exhibits similar RH-dependent microrheological trends as glucose, albeit shifted to a higher $\mathrm{RH}$. That is, there is a gradual increase in $\tau$ as $\mathrm{RH}$ is decreased (approximately 1 order of magnitude increase in $\tau$ for every 5- 


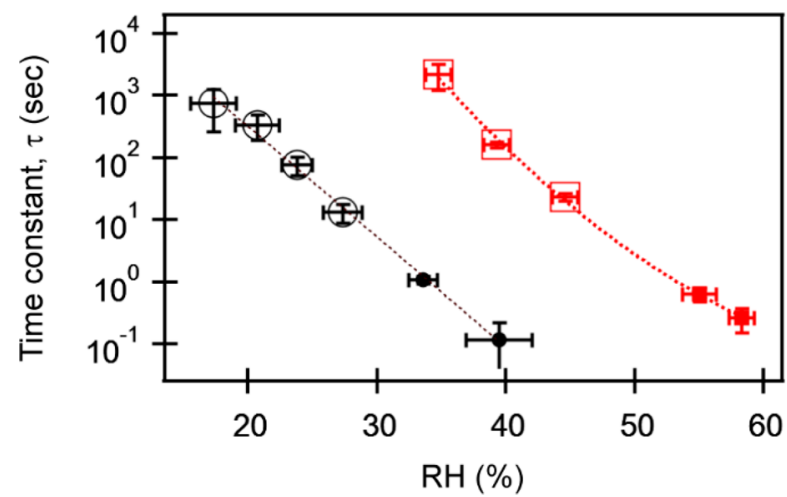

Figure 3. Plot of $\tau$ as a function of $\mathrm{RH}$ for merging events of binary glucose (black circles) and sucrose (red squares). Open markers: data from bright-field aspect ratio. Closed markers: data from far-field imaging. Data points are the average of at least three trials $( \pm 1 \mathrm{SD})$. Dashed lines are polynomial fits.

7\% RH decrease), and every merging event resulted in complete coalescence.

Gel Formation Is Accompanied by an Abrupt Change in Microrheology. The RH-dependent microrheological trends for binary $\mathrm{MgSO}_{4}$ (gel-forming system) are demonstrated in Figure 4. As shown in Figure $4 \mathrm{a}$ at $\sim 35 \% \mathrm{RH}$, merged $\mathrm{MgSO}_{4}$ droplets completely coalesce with a time constant of $\sim 10 \mathrm{~s}$. (This $\tau$-value was $\sim 10 \mathrm{~s}$ regardless of droplet surface charge, position above the counterbalance, and equilibration time, as shown in Figure S4.) If $\mathrm{MgSO}_{4}$ followed expectations from the Newtonian fluids, decreasing the $\mathrm{RH}$ to $30 \% \mathrm{RH}$ would result in an order of magnitude increase in $\tau$. However, as shown in Figure $4 \mathrm{~b}$, upon decreasing the $\mathrm{RH}$ to $30 \%$, merged dimers remain completely rigid and do not coalesce even at long observation times $\left(t_{\mathrm{obs}}\right)$ over $10^{4} \mathrm{~s}$. This demonstrates an abrupt change in microrheology. As shown in Figure 4c, this abrupt change in microstructure is reversible, where increasing the $\mathrm{RH}$ from 30 to $35 \% \mathrm{RH}$ results in complete coalesce. This behavior is consistent with expectations for a gel transition, where the rigid semisolid network assembles and collapses at a relatively welldefined solute concentration. ${ }^{7,32,33}$

As shown in Figure 5 (and Figure S5), the change in RHdependent microrheology of $\mathrm{MgSO}_{4}$ is indeed abrupt relative to the Newtonian fluids (binary glucose and sucrose), with a transition from fluid to rigid gel occurring within an $\mathrm{RH}$ range $<5 \%$, with complete rigidity at $\sim 30 \% \mathrm{RH}$. We note that in Figure $5, t_{\mathrm{obs}}$ is reported when no coalescence was observed (at $30 \%$ $\mathrm{RH}$, which is consistent with previous observations of gel formation). ${ }^{7}$ This value has no physical meaning, and the upper

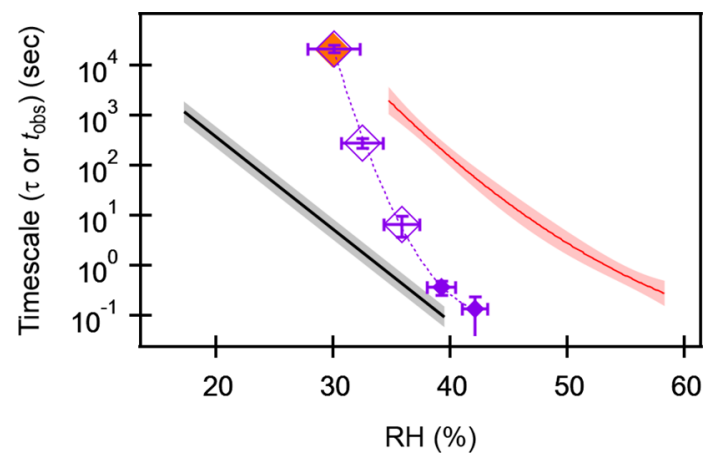

Figure 5. Plot of time scale ( $\tau$ or $t_{\text {obs }}$ ) as a function of $\mathrm{RH}$ for merging events of $\mathrm{MgSO}_{4}$ compared to binary glucose and sucrose. Open triangles: $\tau$ from bright-field aspect ratio. Closed diamonds: $\tau$ from farfield imaging. Orange-filled open diamond indicates rigid gel formation: no coalescence was observed, and $t_{\mathrm{obs}}$ is reported. Data is average of at least three trials $( \pm 1 \mathrm{SD})$. Dashed line is polynomial fit to $\mathrm{MgSO}_{4}$ data. Black and red lines are polynomial fits to, respectively, glucose and sucrose (data from Figure 3; envelopes are 75th percentile confidence bands).

limit was experimental practicality. If coalescence were to ever occur, the associated time constant would be orders of magnitude larger than $t_{\mathrm{obs}}$. However, even without observing at longer time scales, $t_{\mathrm{obs}}$ serves to illustrate the abrupt change in microrheology that occurs with a microgel phase transformation in levitated microdroplets.

Identifying Viscosity and Gel Transitions. DBQ-EDB Microrheological Observations Can Be Used To Understand Aerosol Microstructure. To distinguish between gel-forming systems and Newtonian fluids, we used observations of merged droplets levitated in our recently developed DBQ-EDB in a humidity-controlled environment. Upon merging of two droplets, the shape of the merged dimer deviates significantly from that of a perfect sphere. For Newtonian fluids, merged dimers exponentially relax to a spherical shape to minimize surface energy. The time it takes to relax to sphericity is related to the viscosity $(\eta)$, surface tension $(\sigma)$, and density of the merged droplets as well as the radius $(r)$ of the relaxed sphere. ${ }^{23}$ Above viscosities of $\sim 40 \mathrm{mPa}$ s, such as is the case in the present study (limited by camera imaging frame rate), merged droplets are in the overdamped regime, and relaxation to a spherical shape is characterized by a slow, viscous creeping with no oscillations in shape. ${ }^{23}$ Thus, in the overdamped regime, only the primary oscillation mode needs to be considered, and the characteristic time scale of coalescence $(\tau)$ can be related to viscosity through eq 2
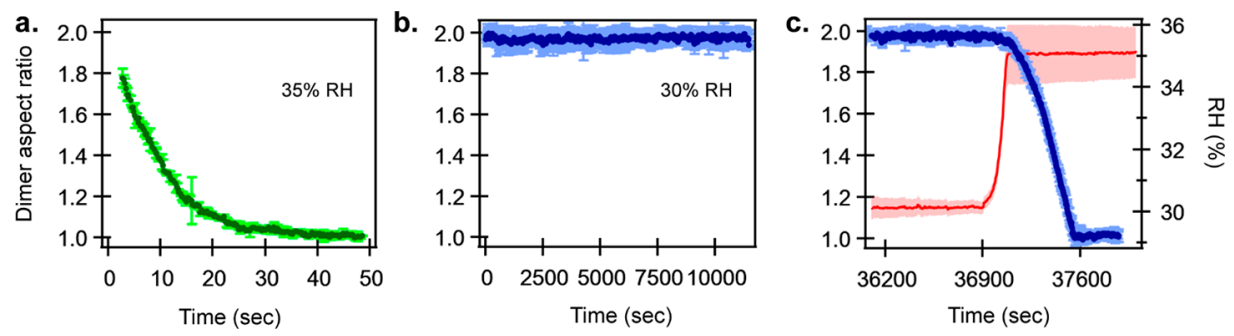

Figure 4. Plots of aspect ratio as a function of time for merging events of $\mathrm{MgSO}_{4}$ droplets. (a) At $35 \% \mathrm{RH}(\tau=10 \mathrm{~s})$. (b) At $30 \% \mathrm{RH}$, merged dimers are completely rigid (evidence for microgel formation). Images were collected continuously for $\sim 12000 \mathrm{~s}$ and then intermittently observed from 12000 to 36000 s. (c) For the same merged dimer shown in part b, the RH was raised from $\sim 30$ to $35 \%$ RH. Gel disassembly and coalescence is observed. For all parts, the average aspect ratio of five sequential images $( \pm 1 \mathrm{SD})$ is plotted. 


$$
\tau \approx \frac{\eta r}{\sigma}
$$

where viscous Newtonian fluids completely coalesce with a single-exponential time constant $\tau$. This is what we observed for binary sucrose and glucose, consistent with expectations and previous observations. ${ }^{18,19,21-23}$

By contrast, microgels do not necessarily ever coalesce due to the semisolid network. ${ }^{9}$ Gels are frequently described as nonNewtonians fluids, i.e., they have different viscoelastic properties depending on the shear stress applied. When shear stress is low or nonexistent, as is the case in the present study, gels can behave like solids below the gelation limit. ${ }^{34}$ Thus, it is not necessarily expected that microgels would flow together, even at long time scales (i.e., $t_{\mathrm{obs}}$ ), consistent with our observations, and it may not be appropriate to describe the gels as having a viscosity. Thus, in the present study, time scales $\left(\tau\right.$ or $\left.t_{\text {obs }}\right)$ were used to differentiate between $\mathrm{RH}$-dependent rheological behavior of glass-forming and gel-forming systems, the former of which can occur gradually over a wide $\mathrm{RH}$ range, ${ }^{23}$ and the latter of which can be characterized by an abrupt phase transition. ${ }^{7,32}$ An abrupt transition is consistent with our observations of $\mathrm{MgSO}_{4}$, as seen in Figure 5.

Aerosol Viscosity Can Be Determined with the DBQ-EDB Technique. Microgel phase transformations have not previously been studied in this manner, so we first establish the validity of our system for determining aerosol viscosity. Sorbitol is a relatively nonviscous monosaccharide and has an established dry viscosity ${ }^{35}$ and was thus used to benchmark the DBQ-EDB and imaging techniques under dry conditions to avoid the additional uncertainty associated with the $\mathrm{RH}$ probes. Using a $30 \mathrm{~min}$ equilibration time under dry conditions ( $1 \pm 2 \% \mathrm{RH})$, a viscosity of $5( \pm 2) \times 10^{4} \mathrm{~Pa} \mathrm{~s}$ was measured for sorbitol (calculated from eq 2 where $\tau=12.7 \pm 4.1 \mathrm{~s}, r=18 \pm 2 \mu \mathrm{m}$, and $\sigma=0.064 \mathrm{~N} \mathrm{~m}^{-1} ; \sigma$ was extrapolated from the parametrization of Yamada et $\mathrm{al}^{36}$ ). This value coincides with the known pure (i.e., dry at $0 \% \mathrm{RH}$ ) sorbitol viscosity of $5 \times 10^{4}$ Pa s. ${ }^{35}$ We further benchmarked the system by determining the RH-dependent viscosity of binary glucose. As shown in Figure S3, our inferred viscosity of glucose is consistent with that reported by Song et al. ${ }^{23}$ (for example, at $40 \% \mathrm{RH}$, we measure a viscosity of $1.5( \pm 0.9) \times 10^{4} \mathrm{~Pa} \mathrm{~s}$, consistent with the $\sim 10^{4} \mathrm{~Pa} \mathrm{~s}$ reported by Song et al. ${ }^{23}$ ). This further demonstrates the validity of the DBQ-EDB for determining the microrheology of microdroplets, including aerosol viscosity.

The RH-Dependent Microrheology of Gels Is Distinct from Newtonian Fluids. Although we have established the capabilities of measuring aerosol viscosity, that was not the intended goal of the present system. Rather, it was to identify gel transitions in aerosol particles and distinguish gel transitions from the gradual increase in viscosity associated with thickening previtrification. To these ends, Figure 5 compares the time scales associated with merging events for $\mathrm{MgSO}_{4}$ (gel-forming compound) to binary saccharides (viscous Newtonian fluids). Over the range of $\mathrm{RH}$ values studied, the overall trend in rheology for binary glucose (and sucrose) is a gradual increase in $\tau$, with an order of magnitude increase for every 5-7\% decrease in $\mathrm{RH}$. As shown in Figure S5, the derivative of $\log (\tau)$ vs $\mathrm{RH}$ remains relatively constant for both glucose and sucrose. This gradual increase in time scale is consistent with viscous Newtonian fluids.

In contrast to the gradual increase in $\tau$ observed with sucrose and glucose, the $\mathrm{RH}$-dependent trend in rheology for gelatinous
$\mathrm{MgSO}_{4}$ is distinct. Specifically, merged $\mathrm{MgSO}_{4}$ droplets exhibit a sharp increase in time scale over a narrow range of $\mathrm{RH}(<5 \%$ $\mathrm{RH})$. As seen in Figure S5, this increase is also reflected in the derivative of the plot of $\log ($ time scale $)$ vs $\mathrm{RH}$. There is an initial increase in $\tau$, coincident with the $\mathrm{RH}$ at which contact-ion pairing is reported to occur $(\sim 35$ to $40 \% \mathrm{RH}) .{ }^{7,8}$ At $\sim 30 \% \mathrm{RH}$, $\mathrm{MgSO}_{4}$ droplets remain completely rigid, consistent with the RH-dependent threshold at which an extensive cross-linked network forms. ${ }^{7}$ This semisolid gelatinous network is apparently rigid enough to prevent coalescence of the merged droplets. As shown in Figure 4c, the rigid gel network disassembles upon increasing $\mathrm{RH}$, consistent with expectations for a phase (gel) transition. ${ }^{32}$ Furthermore, as shown in Figure S4, increasing the equilibration time from 5 to $60 \mathrm{~min}$ did not significantly influence the $\mathrm{RH}$ at which gelation was observed. The distinct difference between the RH-dependent trends for viscous Newtonian fluids (gradual change) versus gelatinous $\mathrm{MgSO}_{4}$ (abrupt transition) demonstrates that gel transitions can be identified using RH-dependent microrheological observations.

$\mathrm{MgSO}_{4}$ Rheological Changes Coincide with Diffusive Limitations. Distinguishing between non-Newtonian gels and Newtonian fluids is relevant, for example, because they exhibit different diffusive limitations. The diffusion coefficient of water $\left(D_{\mathrm{w}}\right)$ within a viscous or gelatinous matrix is an indicator of the rate at which water diffuses through that medium. Knowledge of diffusion rates is important for predicting aerosol reactivity and hygroscopicity. ${ }^{7}$ Measurements of $D_{\mathrm{w}}$ within $\mathrm{MgSO}_{4}$ microgels, as measured by Davies and Wilson, ${ }^{7}$ demonstrate that gelation is accompanied by an abrupt change in $D_{w}$ due to the abrupt formation of the long-range gelatinous network that hinders diffusion. ${ }^{7}$ To further establish that our microrheological observations are consistent with gelation, we compared our reported RH-dependent trends in rheology to measurements of $D_{\mathrm{w}}$ reported elsewhere.

Figure 6 shows the RH-dependent time scales of coalescence, as observed in the present study, plotted with the RH-dependent

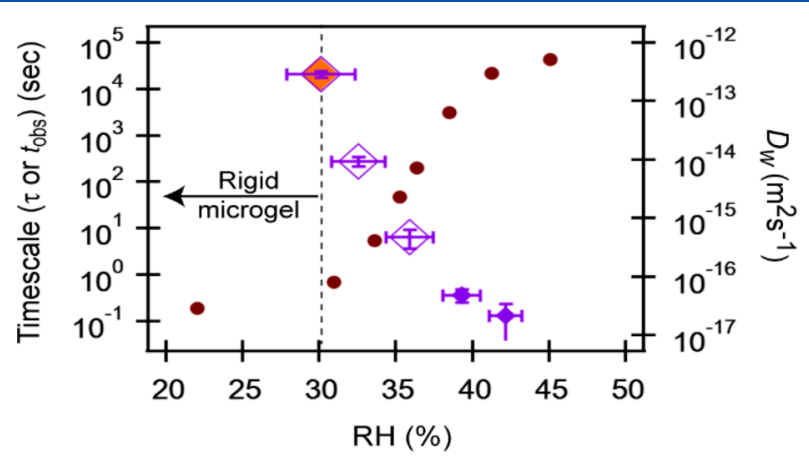

Figure 6. RH-dependent microrheological data (from Figure 5; purple diamonds) plotted along with $D_{\mathrm{w}}$ (red circles). Values for $D_{\mathrm{w}}$ are from Davies and Wilson.

$D_{\mathrm{w}}$ values measured from Davies and Wilson. As seen in Figure 6, Davies and Wilson report gradual changes in $D_{\mathrm{w}}$ below $30 \%$ $\mathrm{RH}$ (after gelation) and above $40 \% \mathrm{RH}$, with an abrupt change in $D_{\mathrm{w}}$ between 30 and $40 \% \mathrm{RH}$. This abrupt change in $D_{\mathrm{w}}$ is coincident with the abrupt change in microrheology of $\mathrm{MgSO}_{4}$, as observed here. Between 30 and $40 \% \mathrm{RH}$, the reported $D_{\mathrm{w}}$ values change by $\sim 4$ orders of magnitude, whereas our measured time scales change by $\sim 5$ orders of magnitude. The greatest diffusive limitation occurs when $\mathrm{MgSO}_{4}$ forms a rigid microgel at $\sim 30 \% \mathrm{RH}$ (where the estimated volume fraction of the pores 
is $\sim 0.3){ }^{7}$ Because we observe rigid gelation at any $\mathrm{RH}$ less than $30 \%$, we cannot use rheological observations to distinguish between expectations of $D_{\mathrm{w}}$ less than $30 \% \mathrm{RH}$ (i.e., merging events at $30 \% \mathrm{RH}$ are indistinguishable from merging events at $25 \% \mathrm{RH})$. However, the coincident changes associated with our rheological observations and reported $D_{\mathrm{w}}$ values suggests that we can infer when the onset of diffusive limitations is to be expected for abrupt aerosol gel transitions.

Gel Transitions Can Be Identified without the Use of Any High-Powered Light Source. The chemical systems studied here are known to minimally absorb visible light and thus are suitable to study with laser sources. ${ }^{7,23}$ However, the ultimate goal of the developed DBQ-EDB will be to apply the technique to studying gel formation involving atmospherically relevant biopolymers and mixtures that may absorb visible light and therefore may have non-negligible heating from a laser source. We thus highlight that the use of far-field laser scatter imaging is not necessary to capture the abrupt transition from fluid to gel for $\mathrm{MgSO}_{4}$. Using just bright-field imaging, which utilizes a gently focused LED (i.e., no laser source) and thus minimizes potential light-induced heating, we captured the transition from fluid at $\sim 35 \% \mathrm{RH}(\tau \approx 10 \mathrm{~s})$ to rigid gel at $30 \% \mathrm{RH}$. Although we demonstrated the use of far-field laser scatter imaging to extend the range of $\tau$-values that we could measure to shorter time scales, this was not necessary in order to detect gelation. The DBQ-EDB technique is therefore suitable for studying gelation of chemical species that strongly absorb visible light, which is an advantage over conventional optical trapping techniques.

Identifying Non-Newtonian Mixtures. Non-Newtonian Flow Is Observed in Mixed Organic-Inorganic Liquid-Solid Suspensions. Non-Newtonian fluid behavior is characterized by nonconstant viscosity at a given temperature. ${ }^{11,37}$ Gels are frequently non-Newtonian. In some instances, gels will behave mechanically like solids under low shear, as illustrated with $\mathrm{MgSO}_{4}$. While we were able to successfully identify the gel transition, we cannot, at present, directly probe whether greater shear stress would induce fluid-like behavior. However, we are able to explore other non-Newtonian behavior exhibited by some gels and many other fluids. ${ }^{11,37}$ Specifically, we explored non-Newtonian behavior in solid-liquid suspensions, a class of fluids that commonly exhibit non-Newtonian behavior. ${ }^{11,37}$

Solid-liquid suspensions and slurries that exhibit nonNewtonian behavior include concentrated suspensions of silicon particles in polyethylene and clay suspensions in water. ${ }^{11,37}$ Droplets of such suspensions cannot be generated in the dispensers used in the present study. Thus, we created solidliquid suspensions in stillo (within the levitated droplet) using model sea spray aerosol compounds composed of 2:1:1 (by mole) $\mathrm{NaCl}: \mathrm{CaCl}_{2}$ :sorbitol microdroplets at $\sim 30 \% \mathrm{RH}$. The initial solution generated from the droplet dispensers is aqueous. As shown in Figure S6a, far-field image analysis of these droplets demonstrates that droplets undergo partial efflorescence at $\sim 30-35 \%$ RH. Further, as shown in Figure S6b, SEM images show that rather than a single, solid crystalline core, the crystalline component is distributed throughout the particle phase as smaller microcrystals. This creates a mixture of solid $\mathrm{NaCl}$ suspended in a viscous fluid (sorbitol/ $\mathrm{CaCl}_{2}$ ), i.e., a slurry. This system was chosen specifically, because we observed the formation of the solid-liquid suspension and because of the natural occurrence of these compounds in sea spray and the environment.

Figure 7 shows the microrheology of merging the partially effloresced liquid-solid droplets. There is an initial, exponential

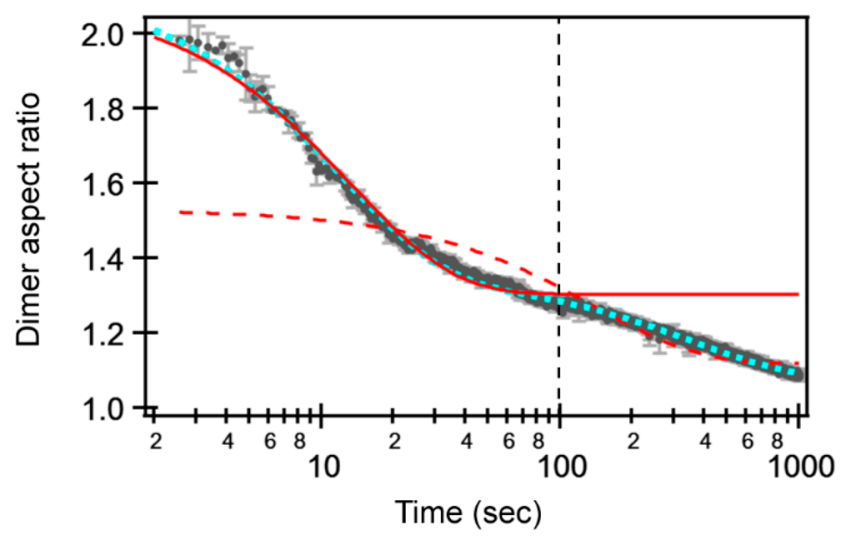

Figure 7. Plot of aspect ratio as a function of time for a single merging event of partially effloresced, mixed-component droplets of 2:1:1 (by mole) $\mathrm{NaCl}: \mathrm{CaCl}_{2}$ :sorbitol. Each data point is the average aspect ratio of five sequential images $( \pm 1 \mathrm{SD})$. The solid red line is the singleexponential fit to the first $100 \mathrm{~s}$ of the data (indicated with vertical dashed line). The dashed red line represents the single-exponential fit to the entire plot. The dashed cyan line is the double-exponential fit to the entire plot.

decrease in aspect ratio from $\sim 2$ to 1.3 over the first $100 \mathrm{~s}$, with an initial time constant of $13.3 \mathrm{~s}$ (if the fit is constrained to the first $100 \mathrm{~s}$; see Figure S7). However, over the remaining $900 \mathrm{~s}$, the decay deviates from single-exponential. Instead, over the next $900 \mathrm{~s}$, there is a gradual decay from 1.3 to $\sim 1$. A singleexponential fit does not capture the entire coalescence process. Rather, a double-exponential fit captures the coalescence process, with $\tau_{1}=12 \mathrm{~s}$ and $\tau_{2}=365 \mathrm{~s}$. This demonstrates that viscosity is not constant over the course of droplet coalescence, i.e., the merged droplets are non-Newtonian fluids, and the technique is sensitive to such.

During the merging event shown in Figure 7, we cannot at present decouple whether the observed non-Newtonian behavior is time-dependent or time-independent, because both time and surface-tension effects are changing simultaneously. In the future, a technique such as diffusing wave spectroscopy could potentially be employed to identify the viscosity of the fluid medium. ${ }^{38}$ However, it is clear that the apparent viscosity increases as the coalescence process progresses, and this is a clear indicator of non-Newtonian fluid dynamics. $^{11,37}$ Thus, with the DBQ-EDB technique, it is possible to identify gel transitions through both the abrupt rheological changes that occur with rigid microgel formation, as demonstrated with $\mathrm{MgSO}_{4}$, as well as non-Newtonian behavior that may occur from in-droplet formation of nonrigid micro and nanogels.

\section{CONCLUSIONS}

We have established here a microanalytical technique that can be used to identify gel formation and non-Newtonian fluids in levitated microdroplets and to measure the viscosity of organic aerosol particles. The DBQ-EDB and described imaging setup is robust, simple to operate, utilizes inexpensive CMOS cameras, and relies on electrostatics for levitation as opposed to laserbased trapping techniques. Additionally, we have described the application of far-field image analysis to track aerosol coalescence using low-power light sources, further simplifying the amount of equipment necessary to study the microrheology of aerosol particles. By eliminating the need for a high-powered trapping laser, this technique will facilitate the study of light- 
absorbing chemical species, which are abundant in atmospherically relevant aerosol particles. Thus, future studies can examine gelation of a wide-range of naturally occurring organic species.

Utilizing this novel microrheological technique, we demonstrated that microgel formation can be distinguished from the gradual thickening associated with vitrification as well as distinguish Newtonian fluids from non-Newtonian fluids. We benchmarked the system with accurate determination of sorbitol and glucose viscosity. This experimental approach can be leveraged to study a wide range of aerosol physicochemical processes relevant to atmospheric science. At present, we are unable to probe for other gel characteristics and material properties, such as yield stress. Further, only RH-dependent transitions can be observed, but temperature-induced transitions could be studied with the addition of a temperaturecontrolled chamber. In its present form, applications of the DBQ-EDB microrheological technique are not limited to environmentally relevant systems, as studied here. The technique can be extended to other biologically, pharmaceutically, and industrially relevant systems. On a fundamental level, the approach can be used to study the rheological characteristics of soft matter materials under a wider range of conditions than is accessible in bulk experiments and thus access novel processes that occur outside of bulk solubility limits.

\section{ASSOCIATED CONTENT}

\section{SI Supporting Information}

The Supporting Information is available free of charge at https://pubs.acs.org/doi/10.1021/acs.analchem.9b04487.

Figure S1. Examples of far-field defect images generated during the far-field image analysis. Figure S2. An example of monitoring counterbalance voltage as a function of time to ensure droplets were at or close to equilibrium. Figure S3. Comparison of coalescence time scale and viscosity for binary glucose with 5 vs. 60 min premerging equilibration times. Figure S4. 5 versus $60 \mathrm{~min}$ equilibration time for $\mathrm{MgSO}_{4}$, and the negligible effect of induction and counterbalance charge. Figure S5. RHdependent slope of plotted time scales as a function of RH. Figure S6. Partial efflorescence of 2:1:1 NaCl:$\mathrm{CaCl}_{2}$ :sorbitol droplets. Figure S7. The initial $100 \mathrm{sec}$ of a merging event of partially effloresced droplets (PDF)

An example of merging droplets while monitoring with bright-field imaging (AVI)

\section{AUTHOR INFORMATION}

\section{Corresponding Author}

Ryan D. Davis - Department of Chemistry, Trinity University, San Antonio, Texas 78212, United States; 10 orcid.org/00000002-4434-1320; Phone: (210)999-7827; Email: rdavis5@ trinity.edu

\section{Authors \\ David S. Richards - Department of Chemistry, Trinity University, San Antonio, Texas 78212, United States \\ Kristin L. Trobaugh - Department of Chemistry, Trinity University, San Antonio, Texas 78212, United States \\ Josefina Hajek-Herrera - Department of Chemistry, Trinity \\ University, San Antonio, Texas 78212, United States}

Complete contact information is available at:

https://pubs.acs.org/10.1021/acs.analchem.9b04487

\section{Author Contributions}

The data was collected and manuscript was written through contributions of all authors.

\section{Notes}

The authors declare no competing financial interest.

\section{ACKNOWLEDGMENTS}

This work was supported by Trinity University and the National Science Foundation (grant AGS-1925208). D.S.R. acknowledges a Murchison Research Fellowship. K.L.T. and J.H-H. acknowledge support from the Welch Foundation (grant W0031). The authors gratefully acknowledge Dr. Michael Jacobs and Dr. Kevin Wilson (Lawrence Berkeley Nat'l Lab) for assistance with the DBQ-EDB, Prof. Jennifer Steele (Trinity) for assistance with SEM imaging, and Prof. Wilson Terrell, Jr., Ryan Hodge, Ernest Romo, and Marc Carpenter for construction and fabrication assistance.

\section{REFERENCES}

(1) Zhang, S.; Ermann, J.; Succi, M.; Zhou, A.; Hamilton, M.; Cao, B.; Korzenik, J.; Glickman, J.; Vemula, P.; Glimcher, L.; Traverso, G.; Langer, R.; Karp, J. Sci. Transl. Med. 2015, 7, 300ra128-300ra128.

(2) Chin, W.-C.; Orellana, M.; Verdugo, P. Nature 1998, 391, 568572.

(3) Kortesuo, P.; Ahola, M.; Kangas, M.; Kangasniemi, I.; Yli-Urpo, A.; Kiesvaara, J. Int. J. Pharm. 2000, 200, 223-9.

(4) Mikhailov, E.; Vlasenko, S.; Martin, S. T.; Koop, T.; Poschl, U. Atmos. Chem. Phys. 2009, 9, 9491-9522.

(5) Marshall, F.; Miles, R.; Song, Y.-C.; Ohm, P.; Power, R.; Reid, J.; Dutcher, C. Chem. Sci. 2016, 7, 1298-1308.

(6) Reid, J.; Bertram, A.; Topping, D.; Laskin, A.; Martin, S.; Petters, M.; Pope, F.; Rovelli, G. Nat. Commun. 2018, 9, 956.

(7) Davies, J.; Wilson, K. Anal. Chem. 2016, 88, 2361-6.

(8) Wang, F.; Zhang, Y.-H.; Li, S.-H.; Wang, L.-Y.; Zhao, L.-J. Anal. Chem. 2005, 77, 7148-7155.

(9) Buajarern, J.; Mitchem, L.; Reid, J. J. Phys. Chem. A 2007, 111, 13038-13045.

(10) Magnin, A.; Piau, J. M. J. Non-Newtonian Fluid Mech. 1990, 36, 85-108.

(11) Chhabra, R. P.; Richardson, J. F. Non-Newtonian Flow and Applied Rheology: Engineering Applications, 2nd ed.; Elsevier: Oxford, UK, 2008.

(12) Jain, S.; Petrucci, G. A. Aerosol Sci. Technol. 2015, 49, 390-399.

(13) Bateman, A. P.; Belassein, H.; Martin, S. T. Aerosol Sci. Technol. 2014, 48, 42-52.

(14) Lee, H.; Ray, K.; Tivanski, A. Anal. Chem. 2017, 89, 1272012726

(15) Ray, K.; Lee, H.; Gutierrez, M.; Chang, F.; Tivanski, A. Anal. Chem. 2019, 91, 7621-30.

(16) Or, V.; Estillore, A.; Tivanski, A.; Grassian, V. Analyst 2018, 143, $2765-2774$.

(17) Song, M.; Liu, P.; Hanna, S.; Zaveri, R.; Potter, K.; You, Y.; Martin, S.; Bertram, A. Atmos. Chem. Phys. 2016, 16, 8817-8830.

(18) Grayson, J.; Song, M.; Sellier, M.; Bertram, A. Atmos. Meas. Tech. 2015, 8, 2463-2472.

(19) Rovelli, G.; Song, Y.-C.; Maclean, A.; Topping, D.; Bertram, A.; Reid, J. Anal. Chem. 2019, 91, 5074-5082.

(20) Marsh, A.; Petters, S.; Rothfuss, N.; Rovelli, G.; Song, Y.; Reid, J.; Petters, M. Phys. Chem. Chem. Phys. 2018, 20, 15086-15097.

(21) Power, R.; Reid, J. Rep. Prog. Phys. 2014, 77, 074601.

(22) Power, R.; Simpson, S.; Reid, J.; Hudson, A. Chem. Sci. 2013, 4, 2597-2604.

(23) Song, Y.; Haddrell, A.; Bzdek, B.; Reid, J.; Bannan, T.; Topping, D.; Percival, C.; Cai, C. J. Phys. Chem. A 2016, 120, 8123-8137.

(24) Fu, P.; Kawamura, K.; Chen, J.; Qin, M.; Ren, L.; Sun, Y.; Wang, Z.; Barrie, L.; Tachibana, E.; Ding, A.; Yamashita, Y. Sci. Rep. 2015, 5, 9845. 
(25) Verdugo, P. Annu. Rev. Mar Sci. 2012, 4, 375-400.

(26) Davies, J.; Miles, R.; Haddrell, A.; Reid, J. Proc. Natl. Acad. Sci. U. S. A. 2013, 110, 8807-8812.

(27) Jacobs, M. I.; Davies, J. F.; Lee, L.; Davis, R. D.; Houle, F.; Wilson, K. R. Anal. Chem. 2017, 89, 12511-12519.

(28) Davies, J. Aerosol Sci. Technol. 2019, 53, 309.

(29) Hart, M.; Sivaprakasam, V.; Eversole, J.; Johnson, L.; Czege, J. Appl. Opt. 2015, 54, F174.

(30) Davis, R. D.; Lance, S.; Gordon, J. A.; Tolbert, M. A. Anal. Chem. 2015, 87, 6186-94.

(31) Davis, R. D.; Tolbert, M. A. Sci. Adv. 2017, 3, No. e1700425.

(32) Li, Y.; Tanaka, T. Annu. Rev. Mater. Sci. 1992, 22, 243-277.

(33) Liu, X. Top. Curr. Chem. 2005, 256, 1-37.

(34) Dawn, A.; Kumari, H. Chem. - Eur. J. 2018, 24, 762-76.

(35) Rothfuss, N.; Petters, M. Environ. Sci. Technol. 2017, 51, 271279.

(36) Yamada, M.; Fukusako, S.; Kawanami, T.; Sawada, I.; Horibe, A. Int. J. Thermophys. 1997, 18, 1483-1493.

(37) Mujumdar, A.; Beris, A.; Metzner, A. J. Non-Newtonian Fluid Mech. 2002, 102, 157-178.

(38) Mason, T. G.; Gang, H.; Weitz, D. A. J. Opt. Soc. Am. A 1997, 14, 139-149. 